\title{
IMPACTOS ECONÓMICOS Y AMBIENTALES DE LAS ESTRATEGIAS DE ALIMENTACIÓN EN LECHERÍAS DE COSTA RICA ${ }^{1}$
}

\author{
Juan Pablo Iñamagua-Uyaguari ${ }^{2}$, Andreas Jenet ${ }^{2}$, Leonardo Gabriel Alarcón-Guerra ${ }^{2}$, \\ Sergio José Vilchez-Mendoza ${ }^{2}$,Francisco Casasola-Coto ${ }^{2}$, Michel A. Wattiaux ${ }^{3}$
}

\section{RESUMEN}

Impactos económicos y ambientales de las estrategias de alimentación en lecherías de Costa Rica. El objetivo de este trabajo fue analizar las prácticas de alimentación en fincas lecheras de Costa Rica y su influencia en las emisiones de gases con efecto invernadero (GEI). Se evaluó metano $\left(\mathrm{CH}_{4}\right)$, óxido nitroso $\left(\mathrm{N}_{2} \mathrm{O}\right)$ y el índice ingresos sobre gastos de alimentación (IOFC). El estudio se llevó a cabo en 104 fincas lecheras, las cuales se distribuyeron en un rango de altitud entre los 1000 y $2400 \mathrm{msnm}$. Mediante un análisis de conglomerados en función de alimentos consumidos, tiempo de animales en pastoreo, áreas destinadas a potreros y pastos de corte. Se identificaron cuatro estrategias de alimentación, las cuales presentaron diferencias en las proporciones de categorías de alimentos que componen la ración diaria. Las emisiones, expresadas como dióxido de carbono equivalente por leche corregida $\left(\mathrm{CO}_{2}\right.$ eq/LCGP), fueron mayores para las estrategias de alimentación altas en forraje y bajas para estrategias en altitudes elevadas. El concentrado representó los mayores costos de alimentación en todas las estrategias, y significó más del $50 \%$ en el sistema de producción basado en el uso intensivo de concentrado; mientras que el costo de los fertilizantes fue más elevado en las producciones a base de pastos. Los ingresos por venta de leche fueron mayores en la estrategia del sistema intensivo. Sin embargo, el IOFC no presentó diferencias entre las estrategias a pesar de las diferencias en producción de leche. Las estrategias de alimentación influyeron en las emisiones de GEI y en los costos de alimentación. Las estrategias asociadas con menores emisiones dentro de la finca fueron las más dependientes de insumos externos.

Palabras claves: ganadería de leche en trópicos, ingresos sobre costos de alimentación, gastos de alimentación, emisiones GEI, metano, óxido nitroso.

\begin{abstract}
Environmental and economic impacts of feeding strategies in Costa Rican dairy farms. The aim of this study was to analyze feeding practices in Costa Rica's dairy farms and their influence on greenhouse gas emissions (GHG). Methane $\left(\mathrm{CH}_{4}\right)$, nitrous oxide $\left(\mathrm{N}_{2} \mathrm{O}\right)$, and income over feed cost (IOFC) were evaluated. This study was carried out at 104 dairy farms, which were distributed within a range of altitude from 1000 to 2400 meters above sea level. Using cluster analysis based on type of feed, grazing period, areas of forage and pastures; four feeding strategies were identified, which included different proportions of feed categories in daily rations. Emissions expressed as carbon dioxide equivalent per kilo of fat and protein corrected milk $\left(\mathrm{CO}_{2}\right.$ eq/FPCM $)$ were higher for forage feeding strategies and lower for farms found in higher altitudes. Concentrate feed accounted for the higher cost in all feeding strategies, and represented more than $50 \%$ in the intensive concentrate based production system, whereas the costs of fertilizers were higher in pasture based feeding strategies. Income from milk sales was higher for the concentrate based feeding strategy. However, IOFC was the same for all feeding strategies in spite of the large differences in daily milk production. Feeding strategies influenced GHG emissions and feeding costs. Feeding strategies associated with lower GHG emissions within the farm were more dependent from external inputs.
\end{abstract}

Keywords: tropical dairy farms, income over feed cost, feeding cost, GHG emissions, methane, nitrous oxide.

\footnotetext{
Recibido: 24 de febrero, 2015. Aceptado: 21 de julio, 2015. Este trabajo forma parte de la tesis del primer autor para optar por el grado de Magister Scientae en Agroforestería Tropical en el Centro Agronómico Tropical de Investigación y Enseñanza- CATIE, Turrialba, Cartago, Costa Rica. Estudio financiado por el programa Enhancing Capacity for Low Emission Development Strategies (EC-LEDS) del Gobierno estadounidense por U.S. Department of Agriculture Federal Grant 58-3148-3-004.

2 Centro Agronómico Tropical de Investigación y Enseñanza, CATIE 7170 Turrialba, Cartago, Costa Rica. jinamagua@catie.ac.cr, a.jenet@ alumni.ethz.ch (autor para correspondencia), lguerra@catie.ac.cr, svilchez@catie.ac.cr, fcasasol@catie.ac.cr

3 Universidad de Wisconsin - Madison, Departamento de Ciencia Láctea. Wisconsis, Estados Unidos. wattiaux@wisc.edu
} 


\section{INTRODUCCIÓN}

Costa Rica en su "Plan Nacional de Desarrollo 2007-2010" expresó su compromiso de asumir el liderazgo en el tema del cambio climático. Este se oficializó con la iniciativa presidencial "Paz con la Naturaleza", en la cual se contempló el cambio climático como un tema de acción prioritario y se asumió el compromiso de que Costa Rica fuera un país carbono neutral para el año 2021 (MINAET, 2009).

El compromiso de Costa Rica de alcanzar la carbono neutralidad por medio de medidas de adaptación y mitigación se ratificó en el Plan Nacional de Desarrollo 2011-2014 y la Política Agroalimentaria y de Desarrollo Rural 2010-2021, (MIDEPLAN, 2010; SEPSA, 2013), bajo las cuales se ha iniciado la construcción de la estrategia nacional de ganadería baja en carbono, mediante la elaboración de las NAMA ganadería (Acciones Nacionales Apropiadas de Mitigación, por sus siglas en inglés) (MAG, 2013).

Con la aplicación de las NAMA ganadería, Costa Rica pretende alcanzar un potencial de mitigación de 12,93 millones de toneladas de $\mathrm{CO}_{2}$ eq en los próximos quince años. Esto mediante el fortalecimiento de la cadena productiva bovina, el sistema MRV (medición, revisión y verificación) de las capacidades en el sector productivo, industrial, institucional, y mediante la vinculación de las NAMA con la estrategia nacional REDD+ (reducción de emisiones por deforestación y degradación forestal) bajo el programa de carbono neutralidad (MAG, 2013).

Para el año 2005, el inventario nacional de emisiones de Costa Rica determinó un total de 12,2 millones de toneladas de $\mathrm{CO}_{2}$ eq. De este total, las emisiones de metano $\left(\mathrm{CH}_{4}\right)$, por fermentación entérica representaban el $15 \%$, las emisiones de $\mathrm{CH}_{4}$ por fermentación entérica provenientes del sector lechero, representaban aproximadamente el 5\% de las emisiones provenientes de actividades agrícolas (Chacón et al., 2009). Mientras que las emisiones de óxido nitroso $\left(\mathrm{N}_{2} \mathrm{O}\right)$ en pasturas, representaban el $18 \%$ del total de emisiones. A nivel general, las emisiones provenientes de la agricultura representaron el 39\% del total de emisiones en $\mathrm{CO}_{2}$ eq (Chacón et al., 2009).

Las emisiones de $\mathrm{CH}_{4}$ por fermentación entérica son una pérdida de energía; según Johnson y Johnson (1995) esta pérdida puede variar de $2-12 \%$ de la energía bruta ingerida. Las emisiones de $\mathrm{CH}_{4}$ se ven influenciadas por la composición de la dieta y las características de los alimentos. Al estimar las emisiones de $\mathrm{CH}_{4}$ en dos tipos de pasturas en Costa Rica, se encontró que la producción diaria de $\mathrm{CH}_{4}$ aumentaba a medida que disminuía la calidad de la pastura (Montenegro y Abarca, 2001). Además, la manipulación de la dieta (composición y la ingesta de alimento) es una de las estrategias de mitigación de emisiones de $\mathrm{CH}_{4}$ por fermentación entérica (Cottle et al., 2011).

Las emisiones de $\mathrm{N}_{2} \mathrm{O}$ en ganadería a nivel global son estimadas en 1,5 Tg. De estas emisiones, el $41 \%$ corresponde a excretas sólidas y líquidas manejadas en pasturas, $27 \%$ a fuentes indirectas, $19 \%$ a los desperdicios de animales manejados en establos, $10 \%$ a la aplicación de desechos en campos y $3 \%$ a la quema de estiércol (Oenema et al., 2005). Las emisiones de $\mathrm{N}_{2} \mathrm{O}$ provenientes de las excretas son resultado de la ineficiencia en el uso del $\mathrm{N}$ consumido, y se considera que del 70-90\% del $\mathrm{N}$ consumido por los herbívoros puede eliminarse a través de las excretas (Schils et al., 2013).

El sector lechero costarricense ha presentado un crecimiento del 19,6\% en el período 2008 - 2013 (Madriz, 2013). Los incrementos en la producción de leche contrastan con la superficie destinada a pastos a nivel nacional, la cual ha disminuido de 1,8 millones de ha en 1990 a 1,3 millones de ha en el 2011 (FAOSTAT, 2014). La disminución de áreas destinadas a pastizales y el incremento de la producción de leche pueden significar que las ganaderías de leche están experimentando procesos de especialización e intensificación, lo que justificaría estos incrementos en la producción.

El compromiso de Costa Rica de ser carbono neutral y el establecimiento de marcos políticos que incluyan estrategias de mitigación de emisiones en ganadería, hacen que sea necesario identificar acciones que contribuyan a entender mejor las emisiones de GEI provenientes de sistemas ganaderos. En este contexto, el objetivo de este trabajo fue analizar las prácticas de alimentación en fincas lecheras de Costa Rica y su influencia en las emisiones de gases con efecto invernadero (GEI).

\section{MATERIALES Y MÉTODOS}

\section{Población de estudio}

Este estudio se realizó en fincas de productores asociados a la Cooperativa Dos Pinos en Costa Rica; esta 
cuenta con 1420 socios, de los cuales, aproximadamente el $81 \%$ corresponde a lechería especializada y el $19 \%$ a sistemas doble propósito (producción de leche y carne). El criterio para la selección de fincas consideró que estas fueran de lechería especializada y que contaran con información completa de ingresos y egresos de la actividad ganadera correspondiente al año 2013. La predisposición de los productores para participar en una encuesta estructurada fue indispensable, con base en estos criterios, se obtuvo un total de 104 fincas sobre las que se realizó el estudio.

A nivel general, las fincas estudiadas están ubicadas en las provincias de Alajuela, Cartago, Guanacaste, Heredia y San José, en altitudes desde los 100 hasta los 2400 msnm y en los pisos climáticos basal, montano bajo y premontano (Ortiz, 2009).

\section{Recolección de datos y descripción de las variables}

La información correspondiente a ingresos y egresos se obtuvo mediante los programas Sical Web, Sical Excel, Infodairy y Herdboss. Esta información fue proporcionada por la Cooperativa Dos Pinos, al igual que las coordenadas espaciales, información de áreas de manejo, producción de leche al día y contenido de proteína y grasa en la leche. El contenido de proteína y grasa en la leche de la Cooperativa Dos Pinos, lo obtiene a partir del muestreo permanente de la leche producida en cada finca, esto permite mantener un eficiente control de calidad en sus productos.

La información referente a variables de ubicación espacial, altitud y piso climático, se obtuvieron mediante la utilización del software ArcMap, al proyectar las coordenadas espaciales de las fincas en los mapas digitales, que constan en el Atlas Digital de Costa Rica (Ortiz, 2009).

En los primeros meses de 2014 se realizó una encuesta estructurada con el fin de registrar información referente a: categorías de manejo de animales en el hato, composición racial del hato, peso vivo promedio de los animales, número de animales y características de la alimentación diaria por categoría (cantidad diaria en la ración de cada alimento), características del manejo de los animales (horas/día en establo y en potrero) y del estiércol, manejo de la fertilización, especies de pastos de corte y de pasto de piso.

Las categorías identificadas en la encuesta fueron: vacas en producción, vacas secas, toros adultos, novillas (12-24 meses) y terneras (0-12 meses), aunque se trabajó solamente con las vacas en producción presentes en el hato; no obstante, la información se recolectó para todas las categorías de los animales. La categoría de vacas en producción presentes en el hato, representa aproximadamente el $80 \%$ del total de $\mathrm{CH}_{4}$ entérico en fincas lecheras (Gollnow et al., 2014).

\section{Estrategia de alimentación}

En este estudio, las estrategias de alimentación se definieron como las decisiones tomadas por el productor para la alimentación diaria de las vacas en producción; en este contexto, la cantidad, calidad y tipo de alimento que se suministró diariamente a los animales fueron variables que ayudaron a determinar las estrategias. Decisiones de manejo, como horas en potrero al día, y áreas destinadas a potreros y pastos de corte, influyen también en la oferta y consumo de materia seca (MS) en forma de pastos, por lo que también se consideraron como variables claves para definir las estrategias de alimentación.

Los alimentos utilizados por los productores se clasificaron en categorías. De acuerdo con Buza et al. (2014), estas fueron: aditivos, concentrados, forraje ofrecido al animal, subproductos y pastos de piso. En la categoría aditivos, se consideraron los suplementos ofrecidos al animal, como sales minerales, grasas y levaduras. En la categoría de concentrados, se incluyeron aquellos reportados por los productores en la encuesta, estos alimentos fueron utilizados para suplir los requerimientos de proteína y de energía del animal y poseen un alto porcentaje de MS (más de $80 \%$ ). Las características químicas de los concentrados se obtuvieron de la información proporcionada por los fabricantes; a nivel general, estas presentaban valores promedio de proteína cruda (PC) de $18,1 \%$ de la MS con una desviación estándar de 10,10 fibra neutro detergente (FND), $15,7 \%( \pm 8,8)$ de la MS y digestibilidad in vitro de la MS (DIVMS) de $85 \%$.

En la categoría forrajes ofrecidos se encontraban los ensilajes, henos comprados y pastos de corte cultivados en la finca, que se ofrecen en fresco a los animales durante el período de permanencia en el establo. La categoría subproductos incluyó aquellos alimentos resultantes de procesos agroindustriales que se utilizan para la alimentación animal, como son: cáscara de piña y de banano, banano, cáscara de yuca y cascarilla de soya. La categoría pastos de piso incluyó todas las especies de pasto que el animal consume ad libitum. 
En este estudio se contabilizaron veinte especies de pastos y se registró desde una hasta siete especies de pastos por finca. Las especies se agruparon en los siguientes géneros: Axonopus, Brachiaria, Cynodon, Digitaria, Festuca, Hermarthria, Ischaemum, Lolium, Panicum, Pennisetum y Setaria. A cada género se le asignó un valor porcentual de representatividad; este se dio en función de las áreas reportadas para cada pasto por el productor, en la encuesta.

El pasto kikuyo (Pennisetum clandestinum) se consideró de forma independiente de los pertenecientes al género Pennisetum, esto debido a la diferencia en el contenido de proteína cruda con el resto de especies de este género.

Para todos los alimentos se obtuvo información de contenido de MS, PC, FND, extracto etéreo (EE) y DIVMS. La información sobre las características de los subproductos y pastos, provino de una revisión bibliográfica de estudios realizados durante los años 1992-2013 por el Centro de Investigaciones en Nutrición Animal-CINA de la Universidad de Costa Rica (Villarreal, 1992; Sánchez y Soto, 1996; Sánchez et al., 1998; Sánchez y Soto, 1999a; Sánchez y Soto, 1999b; Mora y Figueroa, 2005; Soto et al., 2005; Amador et al., 2010; Chacón-Hernández y VargasRodríguez, 2010; Villalobos y Sánchez, 2010; Mata, 2011; Villalobos et al., 2013).

\section{Consumo de materia seca, proteína, fibra y grasa} por vaca por día

Para la estimación del consumo diario de MS se utilizó la metodología propuesta por NRC (2001), en la cual se predice el consumo diario de MS en función del peso vivo, de la producción de leche, porcentaje de grasa en leche y actividades relacionadas con el manejo de los animales. Con el fin de obtener estimaciones de consumo de MS que reflejen de mejor manera el manejo de los productores, se incluyeron factores de energía necesaria para caminar diariamente al ordeño, caminar en potreros, energía para consumir pasto en potreros y energía requerida para la preñez (NRC, 2001). El peso vivo de los animales fue calculado en función de la representatividad de las razas en el hato por el peso reportado para cada raza por el productor.

Para estimar la MS consumida por pastos de piso se siguió la metodología propuesta por Hardie et al. (2014), en la que la diferencia entre la cantidad de MS predicha por NRC y la ofrecida por el productor se asume como la cantidad proveniente de pastos; esta cantidad se distribuyó proporcionalmente a los géneros de pastos existentes en la finca.

En este estudio se calculó también la eficiencia en el uso de los alimentos por parte de las vacas en producción, esto se realizó dividiendo la cantidad de leche producida por día entre el total de MS consumida diariamente. Este índice de eficiencia permite conocer la relación entre consumo de alimento y producción de leche. El cálculo de eficiencia se realizó también para el $\mathrm{N}$ ingerido, donde se dividió el $\mathrm{N}$ presente en la leche entre la cantidad total ingerida.

\section{Emisiones de $\mathrm{CH}_{4}$}

El cálculo de emisiones de $\mathrm{CH}_{4}$ por fermentación entérica se realizó utilizando la metodología propuesta por Dong et al. (2006), la cual calcula las emisiones en función de los requerimientos de energía y digestibilidad de la ración. Así se obtuvo como resultado la cantidad de energía bruta consumida diariamente. Para el cálculo de energía neta requerida para caminar diariamente al ordeño, se asumió como distancia promedio $500 \mathrm{~m}$ de recorrido de potreros a establo, por dos ordeños diarios (PesoVivo*0,00045*1km). El tipo de manejo de los animales (estabulado, semiestabulado, pastoreo) se consideró para calcular la energía neta requerida para ingesta de pastos en potreros, de esta manera, para los animales con manejo semiestabulado se utilizó la mitad del valor recomendado por NRC (2001), mientras que para animales en pastoreo se utilizó el valor total recomendado $\left(0,002 \mathrm{Mcal} / \mathrm{kgPeso} \mathrm{Vivo}^{0,75}\right)$.

\section{Emisiones de $\mathrm{N}_{2} \mathrm{O}$}

La información recolectada en la encuesta permitió identificar las estrategias de fertilización para áreas destinadas a pastos de corte y potreros, de esta manera se registró la aplicación mensual por hectárea de fertilizantes nitrogenados por cada uso del suelo. El nitrógeno $(\mathrm{N})$ total aplicado en cada uso de suelo se obtuvo multiplicando la cantidad aplicada por ha de fertilizante, por el número de meses de aplicación y por el porcentaje de contenido de $\mathrm{N}$.

Las emisiones de $\mathrm{N}_{2} \mathrm{O}$ en fincas provienen de dos fuentes, una orgánica y otra sintética. La fuente orgánica es el estiércol depositado en pasturas, mientras que las emisiones producidas por fuente sintética provienen de la aplicación de fertilizantes nitrogenados en suelos. 
Para las emisiones por fuente orgánica se consideró solamente las excretas durante el tiempo que las vacas permanecen en potreros, se obvió en este estudio las excretas manejadas en el establo, por carecer de información fiable. Las emisiones provenientes de estas excretas se calcularon utilizando la metodología propuesta por Dong et al. (2006), donde se indica que el $2 \%$ del $\mathrm{N}$ de las excretas depositadas en los potreros se emiten en forma de $\mathrm{N}_{2} \mathrm{O}$. La métrica propuesta por los mismos autores exige convertir las emisiones de $\mathrm{N}_{2} \mathrm{O}-\mathrm{N}$ en $\mathrm{N}_{2} \mathrm{O}$, multiplicando las emisiones por 1,57 (44/28). Esta metodología considera el contenido de $\mathrm{N}$ presente en las excretas, el mismo que se obtuvo siguiendo la metodología de balance del $\mathrm{N}$ en los animales, propuesta por Van Horn et al. (1994), donde se asume que el contenido de $\mathrm{N}$ en las excretas es igual a la diferencia entre el $\mathrm{N}$ ingerido en la ración menos el exportado diariamente en leche. El $\mathrm{N}$ ingerido en la dieta fue calculado asumiendo que fue el valor resultante de dividir \%PC entre 6,25 (NRC, 2001), mientras que el $\mathrm{N}$ exportado en la leche fue calculado a partir del contenido de $\mathrm{PC}$ en leche, dividido entre 6,38 (Scott, 1986).

Las emisiones de $\mathrm{N}_{2} \mathrm{O}$ provenientes de fuentes sintéticas se obtuvieron a partir de la aplicación anual en la finca de fertilizantes sintéticos nitrogenados. Los datos de aplicación de estos fertilizantes se obtuvieron a partir de la encuesta realizada. En esta consultó la cantidad de fertilizantes nitrogenados aplicados por hectárea y por mes en dos usos de suelo: en pastos de corte y potreros. Las emisiones de $\mathrm{N}_{2} \mathrm{O}-\mathrm{N}$ corresponden al $1 \%$ del $\mathrm{N}$ aplicado en forma de fertilizantes sintéticos (Dong et al., 2006). Estas emisiones se estandarizaron a $\mathrm{N}_{2} \mathrm{O}$ de la misma manera que se realizó en las emisiones por fuentes orgánicas.

\section{Emisiones en $\mathrm{CO}_{2}$ equivalente $\left(\mathrm{CO}_{2}\right.$ eq $)$ por kilogramo de leche}

Las emisiones de $\mathrm{CH}_{4}$ y $\mathrm{N}_{2} \mathrm{O}$ se estandarizaron a su potencial de calentamiento atmosférico, expresado en emisiones de $\mathrm{CO}_{2}$ para un período de 100 años. El potencial de calentamiento atmosférico se entiende como la relación entre el forzamiento radiativo de un kilogramo de gas de efecto invernadero emitido a la atmósfera, y el de un kilogramo de $\mathrm{CO}_{2}$ en un período de tiempo determinado (Dong et al., 2006). De esta manera, se tiene que el $\mathrm{CH}_{4}$ tiene un potencial de calentamiento de 21, mientras que para el $\mathrm{N}_{2} \mathrm{O}$ el potencial de calentamiento es de 310 en comparación con el $\mathrm{CO}_{2}$. Estos valores son los aceptados por el Instituto Metereológico Nacional, responsable del inventario de emisiones de Costa Rica (Houghton et al., 1996; IMN, 2014).

Los datos de contenido de proteína y grasa en leche muestran una alta heterogeneidad, que está asociada a las características productivas de las razas lecheras, así como por las características de la alimentación. Para obtener mediciones que puedan ser comparables entre sí, se estandarizó la producción de leche a su contenido de proteína y grasa (LCPG). Esto se realizó mediante la ecuación propuesta por la Federación Internacional Lechera (IDF, por sus siglas en inglés), donde:

LCPG $(\mathrm{kg} /$ día $)=$ Producción diaria de leche* $\left(0,1226^{*}\right.$ grasa en leche $+0,0776^{*}$ proteína en leche+0,2534) (IDF, 2010).

\section{Gastos directos de alimentación}

En el presente estudio se definieron los gastos directos de alimentación como la sumatoria de los costos de compra de alimentos y de fertilización de pastos y pastos de corte, utilizados en la alimentación diaria de las vacas en producción (Buza et al., 2014). De esta manera, el costo diario (dólares) de la dieta de las vacas en producción fue el resultado del costo del kg de MS ofrecido diariamente a los animales. Para el caso de subproductos como cáscara de piña y banano, se utilizó el costo del transporte. Esta información se recolectó mediante grupos focales de discusión realizado con los productores que participaron en este estudio.

El valor de la alimentación aportada por los pastos se estimó a partir de los costos de fertilización anual de pastos y pastos de corte. El valor total de fertilización en estas áreas se dividió entre 365 y posteriormente por el número total de vacas en producción en finca. El valor resultante fue el costo por animal por día de alimentación por consumo de pastos. Si bien este valor no refleja el costo del kg de MS de pasto consumida, esta estimación permitió obtener un valor referencial y parcial del costo de pastos consumido por animal diariamente.

El cálculo de los ingresos diarios por venta de leche para las fincas en este estudio se expresó en dólares/día. Para esto se utilizó el precio promedio anual del $\mathrm{kg}$ de leche vendido, dato reportado por la 
Cooperativa Dos Pinos; este valor se multiplicó por la producción promedio por animal diariamente.

Los ingresos sobre costos de los alimentos (IOFC, por sus siglas en inglés) se definen como los ingresos por venta de leche menos los costos de alimentación, y son considerados una medida de ingresos monetarios, en vez de una relación (Wolf, 2010). Este valor puede ser calculado por día o por mes (Wolf, 2010; Hardie et al., 2014). En este estudio se calculó el IOFC diario por animal en producción mediante la siguiente ecuación: IOFC (dólares/vaca lactante) = ingreso diario por venta de leche por vaca lactante - gastos diarios en alimentos de vaca lactante.

\section{Análisis estadísticos}

Para identificar las estrategias de alimentación existentes, se utilizó el análisis de conglomerados agrupando las fincas en función del porcentaje de representatividad de cada tipo de alimento en la dieta, áreas destinadas a potreros y pastos de corte, y el tiempo que los animales pasan diariamente en potreros. Para realizar este análisis se utilizó una matriz de datos estandarizados, para eliminar el efecto de escala de medida.

Se utilizó el método de agrupamiento de Ward, el cual fue escogido porque minimiza la pérdida de información en cada unión de conglomerados y forma grupos homogéneos. La distancia utilizada fue Euclidea (Balzarini et al., 2008) con una matriz de datos estandarizada. Para la identificación de los grupos se inició un corte utilizando la distancia promedio de todo el conglomerado. Si a esa distancia no se evidenciaba diferencia entre grupos, se procedía a identificar nuevas agrupaciones hasta encontrar diferencias. El corte al $60 \%$ de la distancia indicó grupos diferentes.

Para confirmar las diferencias entre los grupos identificados en cada proceso e identificar variables que separaban a los grupos, se aplicó un análisis de la varianza Kruskal-Wallis (Conover, 1999). Este análisis de varianza no paramétrica permite comparar la esperanza de dos o más distribuciones, sin la necesidad de comprobar el supuesto de distribución normal de los términos de error (Balzarini et al., 2008). Los análisis fueron realizados con el paquete estadístico InfoStat versión 2014 (Di Rienzo et al., 2014).

\section{RESULTADOS Y DISCUSIÓN}

Los resultados de la encuesta mostraron que la raza predominante fue la Jersey, con 39,5\%, seguida de las razas Holstein y cruces locales con 30,6 y $24,6 \%$, respectivamente. La producción promedio de leche fue de $14,8 \mathrm{~kg} / \mathrm{vaca} /$ día con una desviación estándar de $5 \mathrm{~kg}$. Estos valores fueron inferiores a los reportados por Jiménez-Castro y Elizondo-Salazar (2014), que indicaron una producción diaria media de $19 \mathrm{~kg}$, con una desviación estándar de $6 \mathrm{~kg}$.

El consumo diario promedio de MS por vaca lactante fue de $16,01 \mathrm{~kg}$, con una desviación estándar de 2,57 kg. Los pastos de piso fueron los que aportaron el $52,3 \pm 18,9 \%$ del consumo total de MS, mientras que los concentrados aportaron el $29,5 \pm 10,6 \%$. Los forrajes ofrecidos (henos, ensilajes, pastos de corte) y los subproductos aportaron un $9,3 \pm 11,3$ y $8,8 \pm 13,1 \%$ del total, respectivamente.

El análisis de conglomerados permitió agrupar las fincas que tenían estrategias de alimentación similares, en función de las variables seleccionadas para construir estas estrategias. Con un criterio de diferenciación de conglomerados al $60 \%$ de la distancia, se identificaron cuatro grupos de fincas estadísticamente distintas entre sí (Figura 1).

\section{Descripción de conglomerados}

Los resultados de la estadística descriptiva, permitió identificar que el conglomerado cuatro presentó la mayor cantidad de fincas (37), en contraste, el conglomerado tres agrupó solamente a ocho fincas, y los conglomerados uno y tres agruparon 24 y 35 fincas, respectivamente (Cuadro 1). En cuanto al tamaño de hato productivo, sobresalió el conglomerado dos con 66 vacas en producción, mientras que el promedio de animales para los conglomerados 1,3 y 4 fue de 57,48 y 48 vacas en producción, respectivamente.

En cuanto al área total de las fincas en estudio, los conglomerados tres y cuatro presentaron las menores áreas con 32,3 y 42,3 ha, mientras que los conglomerados uno y dos presentaron las áreas promedio de las fincas más grandes, con 48 y 57,3 ha, respectivamente. El promedio de altitud de las fincas por conglomerado, ubicó a las del conglomerado cuatro como las de mayores alturas, seguidas de las fincas de los conglomerados dos, tres y uno (Cuadro 1). 


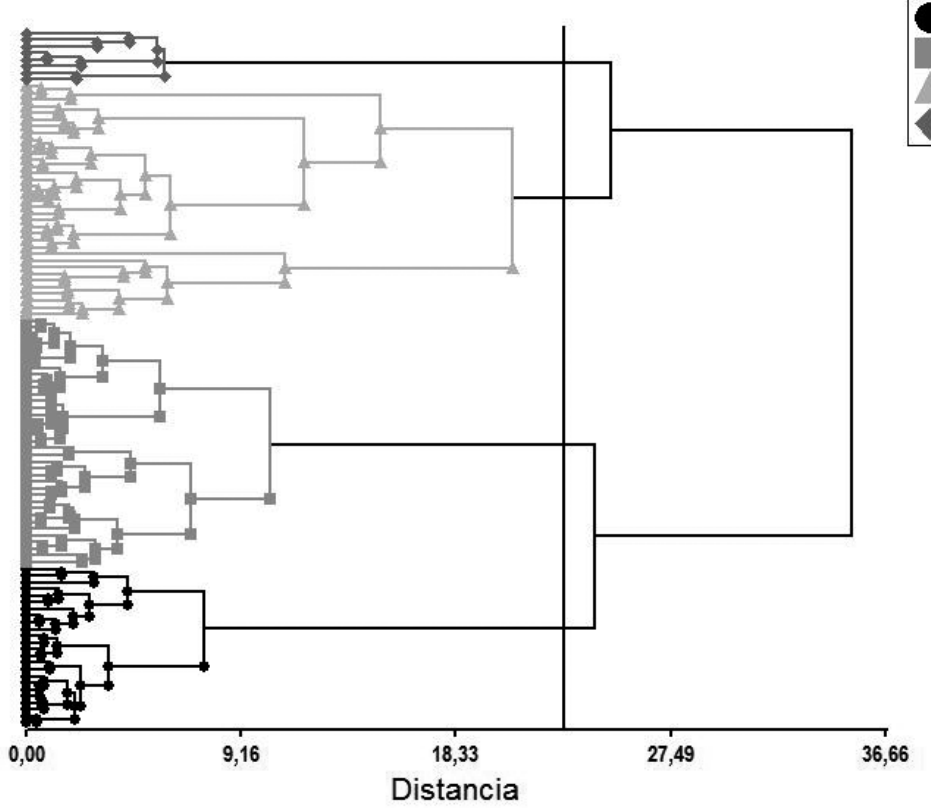

Figura 1. Dendrograma de las fincas lecheras asociadas a la Cooperativa Dos Pinos R.L., agrupado de acuerdo con las estrategias de alimentación basadas en conglomerados en función del porcentaje de representatividad de cada tipo de alimento en la dieta, áreas destinadas a potreros y pastos de corte, y el tiempo que las vacas pasan diariamente en potreros. Costa Rica. 2014.

Figure 1. Dendrogram of dairy farms affiliated to Dos Pinos Cooperative, grouped in clusters according to feeding strategies based on the percentage of type of feed in the diet, areas of forage and cut-and-carry pastures, and the daily time cows spend in pastures. Costa Rica. 2014.

\section{Impactos de las estrategias de alimentación en el consumo y producción}

En este estudio, los cuatro grupos resultantes del análisis de conglomerados se definieron como estrategias de alimentación. Los resultados presentados en el Cuadro 2 muestran que las estrategias difirieron principalmente en el uso de alimentos en la dieta, en el tiempo que los animales pasaban en los potreros y en la superficie destinada a pastos de corte.

La estrategia cuatro que registró el consumo más alto de MS y presentó un mayor porcentaje de animales Holstein en el hato, se caracterizó también por presentar el mayor porcentaje de MS proveniente de concentrados, estadísticamente superior al resto de estrategias (Cuadro 2). Los subproductos ofrecidos, representaron menos del 10\% para las estrategias uno, dos y cuatro, mientras que para la estrategia tres, representó el $46 \%$ de la MS ingerida. La variable forrajes ofrecidos permitió diferenciar a los conglomerados uno y cuatro de los conglomerados dos y tres (Cuadro 2).

El porcentaje de pastos de piso en la dieta presentó diferencias significativas entre los cuatro conglomerados, así se tiene que las fincas del conglomerado uno fueron las que tuvieron un mayor consumo $(70,9 \%)$, seguidas por los conglomerados dos, cuatro y tres con $52,8,47,1$ y $18,7 \%$, respectivamente. La composición de los pastos de piso fue diferente en todas las estrategias, teniéndose que en la estrategia uno los pastos estaban representados por los géneros Brachiaria (40\%), Cynodon (32\%) e Ischaemum (23\%), mientras que en la estrategia dos estuvieron presentes Brachiaria (47\%), Cynodon (27\%), Pennisetum clandestinum (15\%) e Ischaemum (9\%). Los géneros de mayor importancia en la estrategia tres 
Cuadro 1. Características generales de los conglomerados de los hatos lecheros de las fincas lecheras asociadas a la Cooperativa Dos Pinos R.L. y basados en el porcentaje de representatividad de cada tipo de alimento en la dieta, áreas destinadas a potreros y pastos de corte, y el tiempo que las vacas los pasan diariamente en potreros. Costa Rica. 2014.

Table 1. General characteristics of clusters of dairy herds from dairy farms affiliated to Dos Pinos Cooperative, based on the percentage of type of feed in the diet, areas of forage and cut-and-carry pastures, and the daily time cows spend in pastures. Costa Rica. 2014.

\begin{tabular}{lcccr}
\hline Variable & \multicolumn{4}{c}{ Conglomerado } \\
\cline { 2 - 5 } & 1(D.E.) & 2(D.E.) & 3(D.E.) & 4(D.E.) \\
\hline Fincas (N) & 24 & 35 & 8 & 37 \\
Vacas en producción (n) & $56,6( \pm 30,8)$ & $65,9( \pm 47,4)$ & $47,6( \pm 19,8)$ & $47,8( \pm 25,9)$ \\
Raza Holstein (\%) & $15,4( \pm 32,2)$ & $36,5( \pm 38,2)$ & $15( \pm 22,7)$ & $38,3( \pm 40,7)$ \\
Raza Jersey (\%) & $42,3( \pm 41,9)$ & $40,0( \pm 34,1)$ & $41,3( \pm 37,6)$ & $37,0( \pm 37,2)$ \\
Cruces locales (\%) & $31,9( \pm 42,2)$ & $20( \pm 30)$ & $31,3( \pm 35,6)$ & $22,7( \pm 30,5)$ \\
Consumo de materia seca & $15,25( \pm 2,16)$ & $15,42( \pm 2,44)$ & $16,03( \pm 2,22)$ & $17,1( \pm 2,75)$ \\
(kg/día) & & & & $42,3( \pm 26,7)$ \\
Área total (ha) & $48,0( \pm 27,6)$ & $57,3( \pm 46)$ & $32,3( \pm 14,0)$ & $908( \pm 630)$ \\
Altitud (msnm) & $383( \pm 410)$ & $746( \pm 682)$ & $650( \pm 754)$ & $908)$ \\
\hline
\end{tabular}

Cuadro 2. Estrategias de alimentación de las vacas lactantes en las fincas lecheras asociadas a la Cooperativa Dos Pinos R.L. y basadas en conglomerados en función del porcentaje de representatividad de cada tipo de alimento en la dieta, áreas destinadas a potreros y pastos de corte, y el tiempo que las vacas pasan diariamente en potreros. Costa Rica. 2014.

Table 2. Feeding strategies for lactating cows on dairy farms affiliated to Dos Pinos Cooperative grouped in clusters based on the percentage of representativity of type of feed in the diet, areas of forage and pastures, and the daily time cows spend in pastures. Costa Rica. 2014.

\begin{tabular}{|c|c|c|c|c|c|}
\hline \multirow[t]{2}{*}{ Variable } & \multicolumn{4}{|c|}{ Conglomerado } & \multirow[b]{2}{*}{$\mathbf{P}$} \\
\hline & 1(D.E.) & 2(D.E.) & 3(D.E.) & 4(D.E.) & \\
\hline Concentrado $(\%)^{1}$ & $22,9^{\mathrm{A}}( \pm 7,3)$ & $26,2^{\mathrm{A}}( \pm 10,5)$ & $26,3^{\mathrm{A}}( \pm 7,5)$ & $37,7^{\mathrm{B}}( \pm 7,8)$ & $<0,001$ \\
\hline Subproductos $(\%)^{1}$ & $4,1^{\mathrm{A}}( \pm 4,8)$ & $4,4^{\mathrm{A}}( \pm 7,4)$ & $46,0^{\mathrm{C}}( \pm 15,9)$ & $7,8^{\mathrm{B}}( \pm 5,9)$ & $<0,001$ \\
\hline Forraje ofrecido $(\%)^{1}$ & $2,1^{\mathrm{A}}( \pm 3,8)$ & $16,6^{\mathrm{B}}( \pm 14,6)$ & $9,1^{\mathrm{B}}( \pm 9,3)$ & $7,2^{\mathrm{A}}( \pm 7,0)$ & $<0,001$ \\
\hline Pastos de Piso $(\%)^{1}$ & $70,9^{\mathrm{D}}( \pm 8,1)$ & $52,8^{\mathrm{C}}( \pm 17,9)$ & $18,7^{\mathrm{A}}( \pm 17,4)$ & $47,1^{\mathrm{B}}( \pm 10,5)$ & $<0,001$ \\
\hline Horas potrero al día & $20^{\mathrm{B}}( \pm 2,5)$ & $13,1^{\mathrm{A}}( \pm 2,8)$ & $17,6^{\mathrm{B}}( \pm 4,8)$ & $18,4^{\mathrm{B}}( \pm 2,3)$ & $<0,001$ \\
\hline Área Pastos corte (ha) & $0,5^{\mathrm{A}}( \pm 1,0)$ & $3,6^{\mathrm{C}}( \pm 4,9)$ & $2,6^{\mathrm{BC}}( \pm 3,4)$ & $1,2^{\mathrm{AB}}( \pm 1,7)$ & $<0,001$ \\
\hline Área potreros (ha) & $19,8( \pm 9,0)$ & $24,6( \pm 19,9)$ & $16,5( \pm 9,2)$ & $14,8( \pm 6,7)$ & NS \\
\hline
\end{tabular}

${ }^{1}$ : Porcentaje en la MS de la ración / Percentage of dry matter in the ration.

A,B,C,D: Prueba Kruskal-Wallis, valores dentro de una fila que no comparten el mismo superíndice son diferentes $(\mathrm{P}<0,5) / \mathrm{Kruskal}-$ Wallis test, values within a row that do not share a superscript letter differ $(\mathrm{P}<0.05)$.

fueron Brachiaria (49\%), Cynodon (25\%), Ischaemum (13\%) y Pennisetum clandestinum (13\%), mientras que en la estrategia cuatro, la representación de pastos fue la siguiente: Brachiaria (39\%), Cynodon (32\%),
Pennisetum clandestinum (20\%), Ischaemum (3\%) y Lolium con un $2 \%$.

La variable área de pastos de cortes diferenció al conglomerado uno con 0,5 ha del resto de 
conglomerados (Cuadro 2). El conglomerado dos, con 3,6 ha presentó el área más grande destinada a pastos de corte, diferenciándose del conglomerado cuatro con 1,2 ha.

El tiempo que los animales pasan diariamente en potreros identificó como el de menor tiempo en potreros al conglomerado dos con 13,1 horas/día, y fue diferente del resto de conglomerados. El área destinada a potreros no presentó diferencias estadísticas entre los conglomerados, aunque el cuatro presentó las menores áreas promedio, con 14,8 ha, mientras que el de mayor área fue el dos, con 24,6 ha.

El análisis de las características de los alimentos utilizados en cada estrategia de alimentación, permitió identificar que el contenido de PC no presentó diferencias significativas, de esta manera, para todas las estrategias, se registraron valores promedio de $\mathrm{PC}$ de $16,9 \pm 3,47 \%$ de la MS para concentrados, $6,1 \pm 1,6 \%$ de la MS para subproductos, $11,3 \pm 5,1 \%$ de la MS para forrajes ofrecidos y 12,3 $\pm 2,9 \%$ de la MS de pastos de piso. El estudio realizado por Sánchez et al. (1998), en fincas lecheras con una producción diaria promedio de leche de $10 \mathrm{~kg} /$ animal y una fertilización nitrogenada anual promedio de 100 a $125 \mathrm{~kg} / \mathrm{ha}$, en la zona norte de Costa Rica, reportó un valor promedio de 10,6\% de PC para los pastos Cynodon nlemfluensis, Brachiaria ruziziensis e Ischaemum indicum. Esto es similar al promedio encontrado en la estrategia uno, de 11,2\% de PC, con una predominancia de los géneros Cynodon (32\%), Brachiaria (40\%) e Ischaemum (23\%). La estrategia cuatro con una fertilización promedio de $174,6 \mathrm{~kg} / \mathrm{N} / \mathrm{ha} / \mathrm{año}$, reportó un contenido promedio de PC del $13,1 \%$, resultado similar al estudio realizado por Villalobos et al. (2013) en las provincias de Alajuela, Cartago y San José, en fincas lecheras con una fertilización nitrogenada anual promedio de 540 $\mathrm{kg} / \mathrm{ha}$, quienes encontraron un contenido promedio de 13,3\% de PC para los pastos Cynodon nlemfluensis, kikuyo y Lolium perenne.

El contenido de FND presentó valores similares en todas las estrategias para pastos de piso $(67,2 \pm 5,1 \%$ de la MS), concentrados $(14,9 \pm 1,3 \%$ de la MS) y forraje ofrecido, con $67,7 \pm 5,3 \%$ de la MS. En el caso de los alimentos tipo subproductos, el contenido de FND diferenció a las estrategias uno y cuatro con $24,8 \pm 13,9$ y $31,4 \pm 16,5 \%$ de la MS, de las estrategias dos y tres con $43,5 \pm 25,6$ y $37,8 \pm 13,6 \%$ de la MS, respectivamente.

El EE presente en los forrajes ofrecidos no presentó diferencias entre estrategias, teniéndose valores promedio de 0,86, con una desviación estándar de $1,47 \%$ de la MS. En los concentrados, el EE diferenció a la estrategia dos (con $3,2 \pm 2,8 \%$ de la MS) como de menor contenido de EE, en relación con la cuatro $(3,3 \pm 1,4 \%$ de la MS) con un contenido medio de EE, y de la estrategia tres $(4,0 \pm 0,86 \%$ de la MS $)$ como de mayor contenido de EE. La estrategia uno, a pesar de presentar el promedio más alto de EE $(4,7 \%)$, su alta desviación estándar $(4,26)$ la identificó como similar a las cuatro y tres, pero diferente a la estrategia dos.

La digestibilidad de la MS en pastos de piso y pastos de corte presentó diferencias entre las estrategias, la uno $(66 \pm 1,9 \%)$ fue la de menor digestibilidad, diferente de la cuatro $(67,4 \pm 1,6 \%)$ que fue la de mayor digestibilidad. Las estrategias dos y tres presentaron valores similares de digestibilidad de la MS $(67,2 \pm 1,4$ y $64,6 \pm 5,8 \%$ respectivamente).

El contenido de PC en la ración diaria ubicó a la estrategia tres $(10,5 \%)$ como la de menor contenido y diferente del resto de estas, mientras que la estrategia cuatro $(13,5 \%)$ fue la que presentó el mayor contenido (Cuadro 3), sin embargo existe la posibilidad de que este valor pueda ser inferior al contenido real en dietas de fincas lecheras de la Cooperativa Dos Pinos (León, comunicación personal, 2014), aunque experiencias del Centro de Investigación en Nutrición Animal (CINA) de la Universidad de Costa Rica, reportan un $12 \%$ de PC y $44,8 \%$ de FND en dietas de vacas Jersey/ Holstein cruzadas, con una producción diaria de leche de $15 \mathrm{~kg}$ (Rojas, comunicación personal, 2014).

Las estrategias de alimentación que presentaban mayor presencia de animales de la raza Holstein y un mayor porcentaje de concentrados en la dieta (estrategias tres y cuatro), presentaron también los valores más altos de producción de leche, esto en comparación con las estrategias uno y dos. Para establecer una mejor relación entre dieta y producción de leche, se utilizó el valor de eficiencia alimenticia, donde los resultados mostraron que las estrategias dos y cuatro fueron similares entre sí, pero diferentes de la uno, y la estrategia tres fue similar a todas las estrategias. Estos resultados sugieren que los animales en la estrategia dos y cuatro presentaron un mayor aprovechamiento del alimento consumido (en comparación a la estrategia uno). Estos resultados fueron similares a los encontrados por Sehested et al. (2003), quienes al comparar la producción de leche en fincas orgánicas, en donde se probó el efecto de la inclusión de concentrados a diferentes niveles en la dieta de vacas lecheras, encontraron una reducción del 
Cuadro 3. Composición química de la ración, digestibilidad, eficiencia de conversión, y producción de leche en las fincas lecheras asociadas a la Cooperativa Dos Pinos R.L. y basadas en conglomerados en función del porcentaje de representatividad de cada tipo de alimento en la dieta, áreas destinadas a potreros y pastos de corte, y el tiempo que los animales pasan diariamente en potreros. Costa Rica. 2014.

Table 3. Chemical composition of the ration, digestibility, conversion efficiency, and milk production in dairy farms affiliated to Dos Pinos Cooperative grouped in clusters based on the percentage of representativity of type of feed in the diet, areas of forage and cut-and-carry pastures, and the daily time cows spend in pastures. Costa Rica. 2014.

\begin{tabular}{|c|c|c|c|c|c|}
\hline \multirow[t]{2}{*}{ Variable } & \multicolumn{4}{|c|}{ Conglomerado } & \multirow[t]{2}{*}{$\mathbf{P}$} \\
\hline & 1(D.E.) & 2(D.E.) & 3(D.E.) & 4(D.E.) & \\
\hline Proteína cruda $(\% \mathrm{MS})^{1}$ & $12,3^{\mathrm{AB}}( \pm 1,8)$ & $12,9^{\mathrm{B}}( \pm 2,4)$ & $10,5^{\mathrm{C}}( \pm 2,4)$ & $13,5^{\mathrm{A}}( \pm 1,5)$ & 0,001 \\
\hline Fibra Neutro detergente $(\% \mathrm{MS})^{1}$ & $55,2^{\mathrm{A}}( \pm 4,2)$ & $52,3^{\mathrm{A}}( \pm 7,3$ & $40,0^{\mathrm{B}}( \pm 9,9)$ & $44,4^{\mathrm{B}}( \pm 5,1)$ & $<0,001$ \\
\hline Digestibilidad (\% MS) ${ }^{1}$ & $70,0^{\mathrm{C}}( \pm 1,6)$ & $71,1^{\mathrm{B}}( \pm 2,3)$ & $75,7^{\mathrm{A}}( \pm 2,3)$ & $73,3^{\mathrm{A}}( \pm 1,7)$ & $<0,001$ \\
\hline Eficiencia consumo alimento & $0,81^{\mathrm{B}}( \pm 0,16)$ & $0,91^{\mathrm{A}}( \pm 0,17)$ & $0,86^{\mathrm{AB}}(0,14)$ & $0,96^{\mathrm{A}}( \pm 0,17)$ & $<0,01$ \\
\hline Eficiencia en el uso del $\mathrm{N}(\%)^{2}$ & $21,6( \pm 4,6)$ & $22,2( \pm 3,1)$ & $27,5( \pm 6,5)$ & $23,0(3,7)$ & NS \\
\hline LCPG $(\mathrm{kg} / \mathrm{d} \text { ía })^{3}$ & $12,7^{\mathrm{B}}( \pm 3,8)$ & $14,1^{\mathrm{B}}( \pm 4,6)$ & $14,1^{\mathrm{AB}}( \pm 3,5)$ & $16,6^{\mathrm{A}}( \pm 4,8)$ & $<0,1$ \\
\hline Proteína en leche $(\%)$ & $3,28^{\mathrm{A}}( \pm 0,13)$ & $3,18^{\mathrm{B}}( \pm 0,14)$ & $3,32^{\mathrm{A}}( \pm 0,14)$ & $3,28^{\mathrm{A}}( \pm 0,25)$ & $<0,1$ \\
\hline Grasa en leche $(\%)$ & $4,11^{\mathrm{A}}( \pm 0,26)$ & $3,95^{\mathrm{B}}( \pm 0,21)$ & $4,14^{\mathrm{AB}}( \pm 0,34)$ & $4,02^{\mathrm{AB}}( \pm 0,30)$ & $<0,1$ \\
\hline
\end{tabular}

${ }^{1}$ Porcentaje en la MS de la ración / Percentage of dry matter in the ration.

${ }^{2}$ Eficiencia en el uso del $\mathrm{N}=100 \mathrm{x}$ [nitrógeno en la leche $(\mathrm{g} / \mathrm{d})$ dividido por nitrógeno ingerido $(\mathrm{g} / \mathrm{d})$ ] / $\mathrm{N}$ use efficiency $=100 \mathrm{x}$ [Nitrogen in milk $(\mathrm{g} / \mathrm{d})$ divided by ingested Nitrogen $(\mathrm{g} / \mathrm{d})$ ]

${ }^{3}$ LCGP $=$ Leche corregida a su contenido de proteína y grasa por IDF (2010) / FPCM = Fat and protein corrected milk according to IDF (2010).

A, B, C, D Prueba Kruskal-Wallis; valores dentro de una fila que no comparten el mismo superíndice son diferentes / Kruskal-Wallis test; values within a row that do not share a superscript letter differ $(\mathrm{P}<0,05)$.

rendimiento de 19 y $8 \%$ en los grupos alimentados con 0 y $19 \%$ de MS en la dieta aportada por concentrados. Esto en comparación con el grupo en el que los concentrados representaban el $38 \%$ de la MS de la dieta.

Dietas con mayor uso de concentrado presentaron un mayor porcentaje de PC en la leche (Bargo et al., 2002; Sehested et al., 2003). En el estudio realizado por Sehested et al. (2003), se reportó mayor contenido de PC en leche en las estrategias con mayor porcentaje de concentrados en la dieta $(3,39$ y $3,36 \%$ de PC, en dietas con 38 y $19 \%$ con concentrados), en comparación con la dieta sin concentrados, la cual presentó 3,28\% de PC en leche. En este estudio, el porcentaje de PC en leche diferenció a la estrategia dos con $3,18 \pm 0,14 \%$ de PC en leche, como la de menor $\mathrm{PC}$, mientras que las estrategias uno, tres y cuatro no presentaron diferencias significativas, con $3,28 \pm 0,13$, $3,32 \pm 0,14$ y $3,28 \pm 0,25 \%$ de PC, respectivamente. Los valores reportados por Sehested et al. (2003) de porcentaje de grasa en leche, mostraron una tendencia distinta a la PC, en donde la dieta de mayor consumo de concentrados (38\% de la MS en la dieta) presentó el menor contenido de grasa $(4,11 \%)$; este resultado es similar al reportado en este estudio, donde la estrategia uno (menor consumo de concentrado) presentó el porcentaje más alto de grasa en leche $(4,11 \pm 0,26 \%)$.

En cuanto al contenido de FND en la dieta, este separó a las estrategias uno y dos, de la tres y cuatro, este resultado podría estar asociado al alto porcentaje de pastos en estas dos dietas, mientras que en las estrategias tres y cuatro, los pastos de piso tienen menor representatividad (Cuadro 2).

La eficiencia en el consumo de alimento parece estar relacionada con el contenido de PC en la dieta, y este a su vez, a la presencia de concentrados, puesto que las estrategias que presentaron el mayor contenido de PC también presentaron mayor eficiencia en el uso del alimento. A pesar de que la estrategia tres reportó el menor contenido de PC en la dieta, presentó una eficiencia alimenticia similar al resto. Esta eficiencia podría estar asociada a la digestibilidad de la MS de la dieta, pues se encontró que esta estrategia presentó la 
mayor digestibilidad, con $75,7 \pm 2,3 \%$ de la MS, superior al resto de ellas, seguida por la cuatro con $73,3 \pm 1,7 \%$, la dos con $71,1 \pm 2,3 \%$ y con la menor digestibilidad, la estrategia uno, con $70 \pm 1,6 \%$ de la MS.

Las características nutricionales descritas tuvieron una influencia directa en la producción de leche, es así que se tiene que las fincas de la estrategia cuatro presentaron la mayor producción de leche, con una producción diaria promedio de 16,6 kgLCPG/ animal, mientras que el resto de ellas presentaron valores estadísticamente similares con 12,7, 14,12 y 14,14 kgLCPG/animal/día para las fincas de las estrategias uno, dos y tres, respectivamente. La eficiencia alimenticia diferenció a las estrategias dos y cuatro $(0,91$ y 0,96$)$ de la uno $(0,81)$, mientras que la estrategia tres presentó valores similares a todas las estrategias con 0,86 (Cuadro 3). La proteína en la leche diferenció a la estrategia dos (menor contenido) del resto de ellas, mientras que el porcentaje de grasa en la leche diferenció a la estrategia uno de la dos. Las estrategias tres y cuatro presentaron contenidos similares de grasa (Cuadro 3).

En cuanto a la eficiencia en el aprovechamiento del $\mathrm{N}$ ingerido, la estrategia tres presentó la mayor eficiencia, con un $27,5 \%$, se diferenció del resto de estrategias, las cuales presentaron valores de 21,6\% (uno), 22,2\% (dos) y $23,0 \%$ (cuatro). Esta eficiencia puede evidenciarse en la cantidad de $\mathrm{N}$ presente en las excretas, donde se tuvo que las estrategias que presentaron mayor eficiencia presentaron un contenido más bajo de nitrógeno en las excretas. De esta manera, la cuatro tuvo mayor contenido de $\mathrm{N}$ presente en excretas con 285,3 g/animal/día, lo cual difiere del resto, que presentaron un contenido de 250,8 , 235,5 y $200,1 \mathrm{~g} /$ animal/día para las estrategias dos, uno $\mathrm{y}$ tres, respectivamente.

En el presente estudio se diferenció las excretas manejadas en el establo de las depositadas en los potreros, mientras los animales se encontraban en ellos. De esta manera, la cantidad de nitrógeno presente en las excretas y depositado en los potreros, dependerá también de las horas que los animales permanecen en los potreros. Así las fincas de las estrategias cuatro y uno presentaron valores de 219 y 196,8 g/animal/día de N proveniente de excretas depositadas en pasturas, valores estadísticamente diferentes de las estrategias tres y dos, que presentaron una deposición de $\mathrm{N}$ por excreta en pasturas de 150,8 y 133,8 g/animal/día, respectivamente.

El análisis de la varianza univariada no encontró diferencias estadísticas en la cantidad de $\mathrm{N}$ sintético aplicado en pastos de piso y pastos de corte; sin embargo, las fincas que presentaron una mayor área destinada a pastos de piso (estrategia dos, Cuadro 1) fueron aquellas que mostraron una mayor aplicación de fertilizantes por hectárea y por año $(211,1 \mathrm{~kg})$, seguidas por las fincas de las estrategias cuatro, uno y tres con $174,6,146,7$ y $112,2 \mathrm{~kg} \mathrm{~N} / \mathrm{ha} /$ año, respectivamente. Esta tendencia se mantuvo en fertilización de áreas de pasto de corte, las estrategias tres y dos que presentaban las áreas más grandes, también presentaron los valores más altos en fertilización (105,7 y 101,9 kgN/ha/año, respectivamente). Las fincas de la estrategia cuatro aplicaron $101,4 \mathrm{kgN} / \mathrm{ha} / \mathrm{año}$, mientras que las de la uno aplicaron la menor cantidad de $\mathrm{N}$ en pastos de corte $(38,1 \mathrm{~kg} / \mathrm{ha} / \mathrm{año})$.

\section{Impactos de las estrategias de alimentación en las emisiones de $\mathrm{CH}_{4}$ y $\mathrm{N}_{2} \mathrm{O}$}

En el análisis de emisiones parciales de GEI por fincas ( $\mathrm{kg} \mathrm{CO}_{2}$ eq/LCPG) (Cuadro 4), las emisiones de $\mathrm{CH}_{4}$ representaron el $68,4,71,2,72,5$ y $67,8 \%$ para las estrategias uno, dos, tres y cuatro, respectivamente. En promedio, las emisiones de $\mathrm{CH}_{4}$ representaron una pérdida de energía correspondiente al 6,5\%, similar a lo reportado por (Dong et al., 2006). Las emisiones de $\mathrm{CH}_{4}$ se ven influenciadas por la cantidad de materia seca consumida, producción de leche y peso vivo del animal (Yan et al., 2006).

A nivel general, las emisiones diarias de $\mathrm{CH}_{4}$ por vaca en producción no presentaron diferencias significativas entre las estrategias. Las fincas que presentaban mayor consumo diario de MS (13,8 $\mathrm{kg}$ ), mayor peso vivo de los animales (450 kg) y mayor producción diaria de leche $(20 \mathrm{~kg})$, presentaron mayores emisiones de $\mathrm{CH}_{4}$ (470 g/animal/día), en comparación con las fincas con consumos de MS de 10,8 y $11,2 \mathrm{~kg}$, peso vivo de 350 y $400 \mathrm{~kg}$ y producción diaria de leche de 15 y $17 \mathrm{~kg}$, que mostraron emisiones diarias de $\mathrm{CH}_{4}$ de 388 y $463 \mathrm{~g} /$ animal, respectivamente (Mora, 2001). En este estudio, aunque se identificó un comportamiento en las emisiones similares, estas fueron inferiores a las reportadas por Mora (2001), pero las estrategias uno, dos y cuatro, presentaron valores superiores a los reportados por Montenegro y Abarca (2001) y IMN (2014), con valores de 249 y 232,9 g/animal/día, respectivamente.

Las características de la dieta, como contenido de fibra y tipo de carbohidratos, pueden influir en 
las emisiones de $\mathrm{CH}_{4}$ (Johnson y Johnson, 1995; NRC, 2001; Patel et al., 2011). En este estudio no se encontró una relación directa entre emisiones de $\mathrm{CH}_{4}$ y características de la dieta, pero sí se encontró una relación directa entre producción de leche y características de la dieta (Cuadro 3).

El $\mathrm{N}_{2} \mathrm{O}$ proveniente de excretas manejadas en pasturas, identificó a la estrategia dos como de menores emisiones diarias, diferente de la uno y cuatro, que presentaron emisiones diarias más altas, con 6,2 y $6,9 \mathrm{~g} / \mathrm{N}_{2} \mathrm{O}$ por vaca lactante (Cuadro 4). La estrategia tres presentó emisiones similares a la uno y dos, pero menores a la cuatro. En el caso de las emisiones de $\mathrm{N}_{2} \mathrm{O}$ provenientes de excretas manejadas en pasturas, estas estuvieron relacionadas directamente con el tiempo que pasaron los animales diariamente en los potreros, así como con el contenido de $\mathrm{N}$ en las excretas. El N presente en excretas varió en las estrategias, y se tuvieron cantidades de 200, 235,5, 250,8 y $285,4 \mathrm{~g} / \mathrm{N} / \mathrm{animal} /$ día, para las estrategias uno, dos, tres y cuatro, respectivamente. El N presente en las excretas puede variar de 200 a 550 g/animal/día (Bannink et al., 2013).

La estrategia de alimentación influyó en las emisiones de $\mathrm{N}_{2} \mathrm{O}$, así, en la dos, que presentó las menores emisiones, las vacas en producción pasaron el

Cuadro 4. Emisiones de gases de efecto invernadero en las fincas lecheras asociadas a la Cooperativa Dos Pinos y basadas en conglomerados en función del porcentaje de representatividad de cada tipo de alimento en la dieta, áreas destinadas a potreros y pastos de corte, y el tiempo que los animales pasan diariamente en potreros. Costa Rica. 2014.

Table 4. Greenhouse gas emissions from dairy farms affiliated to Dos Pinos Cooperative grouped in clusters based on the percentage of type of feed in the diet, areas of forage and cut-and-carry pastures, and the daily time cows spend in pastures. Costa Rica. 2014.

\begin{tabular}{|c|c|c|c|c|c|}
\hline \multirow[t]{2}{*}{ Variable } & \multicolumn{4}{|c|}{ Conglomerado } & \multirow[t]{2}{*}{$\mathbf{P}$} \\
\hline & 1(D.E.) & 2(D.E.) & 3(D.E.) & 4(D.E.) & \\
\hline \multicolumn{6}{|l|}{ Emisiones } \\
\hline $\mathrm{CH}_{4}{ }^{1}$ (g/día) & $267,0( \pm 47,9)$ & $264,6( \pm 51,5)$ & $247,5( \pm 38,2)$ & $292,2( \pm 61,1)$ & NS \\
\hline $\mathrm{N}_{2} \mathrm{O} \operatorname{excretas}^{2}(\mathrm{~g} / \mathrm{día} / \mathrm{v})$ & $6,2^{\mathrm{AB}}( \pm 1,8)$ & $4,2^{\mathrm{C}}( \pm 1,4)$ & $4,7^{\mathrm{BC}}( \pm 2,5)$ & $6,9^{\mathrm{A}}( \pm 1,9)$ & $<0,001$ \\
\hline $\mathrm{N}_{2} \mathrm{O}$ fertilización sintética pastos piso ${ }^{3}(\mathrm{~g} /$ día/ha) & $6,4( \pm 4,8)$ & $9,1( \pm 7,4)$ & $4,9( \pm 3,6)$ & $7,6( \pm 8,2)$ & NS \\
\hline $\mathrm{N}_{2} \mathrm{O}$ fertilización sintética pastos corte ${ }^{3}(\mathrm{~g} /$ día/ha) & $1,6^{\mathrm{B}}( \pm 4,5)$ & $4,40^{A}( \pm 5,6)$ & $4,63^{\mathrm{AB}}( \pm 5,1)$ & $4,35^{\mathrm{B}}( \pm 11,8)$ & 0,0015 \\
\hline $\mathrm{CH}_{4} / \mathrm{LCPG}^{4}(\mathrm{~g} / \mathrm{kg})$ & $21,9^{A}( \pm 3,8)$ & $19,7^{\mathrm{B}}( \pm 3,4)$ & $18,01^{\mathrm{B}}( \pm 2,9)$ & $18,0^{\mathrm{B}}( \pm 1,9)$ & $<0,001$ \\
\hline \multicolumn{6}{|l|}{ Emisiones ( $\left.\mathrm{kg} \mathrm{CO}_{2} \mathrm{eq} / \mathrm{kg} \mathrm{LCPG}\right)$} \\
\hline $\mathrm{CH}_{4}$ & $0,46^{\mathrm{A}}( \pm 0,08)$ & $0,41^{\mathrm{B}}( \pm 0,07)$ & $0,38^{\mathrm{B}}( \pm 0,06)$ & $0,38^{\mathrm{B}}( \pm 0,04)$ & $<0,001$ \\
\hline $\mathrm{N}_{2} \mathrm{O}$ excretas & $0,16^{\mathrm{A}}( \pm 0,05)$ & $0,10^{\mathrm{C}}( \pm 0,03)$ & $0,11^{\mathrm{BC}}( \pm 0,05)$ & $0,13^{\mathrm{AB}}( \pm 0,03)$ & $<0,001$ \\
\hline $\mathrm{N}_{2} \mathrm{O}$ fertilización sintética & $0,06( \pm 0,05)$ & $0,08( \pm 0,09)$ & $0,04( \pm 0,04)$ & $0,06(0,08)$ & NS \\
\hline Emisión parcial $^{5}$ & $0,68^{\mathrm{A}}( \pm 0,15)$ & $0,59^{\mathrm{B}}( \pm 0,14)$ & $0,53^{\mathrm{B}}( \pm 0,10)$ & $0,57^{\mathrm{B}}( \pm 0,11)$ & $<0,01$ \\
\hline
\end{tabular}

${ }^{1}$ Emisiones de metano $\left(\mathrm{CH}_{4}\right)$ entérico / Enteric methane $\left(\mathrm{CH}_{4}\right)$ emission.

${ }^{2}$ Emisiones de óxido nitroso $\left(\mathrm{N}_{2} \mathrm{O}\right)$ por excretas depositadas en potreros / Nitrous oxide emissions $\left(\mathrm{N}_{2} \mathrm{O}\right)$ originated from excreta deposited in pastures.

${ }^{3}$ Emisiones de $\mathrm{N}_{2} \mathrm{O}$ por hectárea por aplicación de fertilizantes sintéticos / $\mathrm{N}_{2} \mathrm{O}$ emission per hectare from syntetic fertilizer application.

${ }^{4}$ LCGP = Leche corregida a su contenido de proteína y grasa por IDF (2010) / FPCM = Fat and protein corrected milk according to IDF (2010).

${ }^{5}$ Suma de las emisiones calculadas, expresadas en $\mathrm{CO}_{2}$ eq/LCPG / Sum of calculated emissions, expressed in carbon dioxide equivalent per milk produced $\left(\mathrm{CO}_{2}\right.$ eq $/ \mathrm{kg}$ FPCM).

A, B, C, D: Prueba Kruskal-Wallis; valores dentro de una fila que no comparten el mismo superíndice son diferentes / Kruskal-Wallis test; values within a row that do not share a superscript letter differ $(\mathrm{P}<0.05)$. 
menor tiempo en potreros, a pesar de que el contenido de $\mathrm{N}$ en la dieta fue similar a las estrategias uno y tres, pero inferior a la cuatro.

A pesar de que la emisión de $\mathrm{N}_{2} \mathrm{O}$ proveniente de la fertilización sintética de pastos de piso no presentó diferencias entre estrategias, la fertilización de pastos de corte diferenció a la uno y cuatro de la dos, mientras que la tres presentó emisiones similares al resto de estrategias. En cuanto a las emisiones de $\mathrm{N}_{2} \mathrm{O}$ provenientes de la fertilización sintética, se pudo evidenciar que esta fue influenciada por el área destinada a potreros y pastos de corte, pues las fincas que presentan mayores áreas, fueron también las que presentaban una mayor aplicación de fertilizantes nitrogenados.

Las emisiones de $\mathrm{CH}_{4}$, expresadas en gramos de $\mathrm{CH}_{4} / \mathrm{kg}$ LCPG, identificaron a la estrategia uno como la de mayor producción de $\mathrm{CH}_{4}$ por kilogramo de LCPG, con una media de $21,9 \mathrm{~g}$, lo que la diferencia del resto de estrategias de alimentación (Cuadro 4).

Las emisiones estandarizadas a $\mathrm{CO}_{2}$ eq/LCGP de $\mathrm{CH}_{4}$, diferencian a la estrategia uno del resto. En el caso del $\mathrm{N}_{2} \mathrm{O}$ proveniente de excretas, este identificó a la estrategia uno como la de mayores emisiones, y a la dos de menores emisiones. La estrategia tres presentó emisiones similares a la dos y cuatro, mientras que la cuatro presentó emisiones similares a uno y tres (Cuadro 4). Las emisiones parciales en $\mathrm{CO}_{2}$ eq/LCPG identificaron a la estrategia uno como la de mayores emisiones, diferentes al resto de estrategias (Cuadro 4). Los resultados obtenidos por Van Middelaar et al. (2014) mostraron como el incremento en la productividad de leche tiene la capacidad de reducir emisiones, ya que las $\mathrm{CH}_{4}$ por animal se diluyen en una mayor producción de leche. En este contexto, se puede observar que la estrategia cuatro, que presentó mayor consumo de concentrados, y a pesar de presentar una mayor emisión de $\mathrm{CH}_{4} /$ animal/día, cuando estas emisiones se expresan en $\mathrm{gCH}_{4} / \mathrm{LCPG}$, presentó un valor de $18 \pm 1,9 \mathrm{kgCH}_{4} / \mathrm{LCPG}$, distinto de la estrategia uno, pero similar a la dos y tres (Cuadro 4).

Impactos de las estrategias de alimentación en la intensidad de emisiones y desempeño económico para un futuro NAMA en lecherías costarricenses

Las fincas lecheras de Costa Rica se caracterizan por ser lecherías de pastoreo. Sin embargo, este estudio demostró que existen distintos grados de dependencia respecto al uso de este componente en la constitución de las estrategias de alimentación y que incide en la intensidad de emisiones GEI en la producción de leche. Fincas pertenecientes a la estrategia uno, mostraron una mayor dependencia de pastos de pisos $70 \%$ (Cuadro 2), con un mayor contenido de fibra $55 \%$ MS en la composición de la dieta y una menor eficiencia en la transformación de MS en litros de leche con 0,81 kg LCPG/kg MS ingerida (Cuadro 3). Esto puede explicar que fuera la estrategia de mayor intensidad de emisiones de $\mathrm{CH}_{4} 21,9 \mathrm{~g} \mathrm{CH}_{4} / \mathrm{kg} \mathrm{LCPG}$ (Cuadro 4). En cambio, fincas lecheras pertenecientes a la estrategia cuatro, tuvieron una menor dependencia de pastos de piso $47 \%$ (Cuadro 2) un menor contenido de fibra en la composición de la dieta 44\% MS (Cuadro 3) y una mayor eficiencia en la transformación de MS en litros de leche con $0,96 \mathrm{~kg} \mathrm{LCPG} / \mathrm{kg}$ MS ingerida, en consecuencia, una menor intensidad de emisiones de $\mathrm{CH}_{4}$ con 18,0 $\mathrm{g} \mathrm{CH}_{4} / \mathrm{kg} \mathrm{LCPG}$ en la producción de leche (Cuadro 3). No obstante, estas diferencias de desempeño ambiental a nivel de emisiones GEI no se relacionaron con un mejor desempeño económico (Cuadro 5).

El indicador IOFC no mostró diferencias significativas entre estrategias de alimentación. Sin embargo, las fincas que tuvieron un mayor consumo de concentrados (estrategia cuatro) presentaron también el mayor valor de IOFC. Este resultado es similar al reportado por Hardie et al. (2014) en un estudio realizado en fincas lecheras de producción orgánica en Wisconsin, USA, donde identificaron que las fincas con mayor IOFC, fueron las que presentaron un mayor consumo diario de concentrados.

El análisis de los gastos directos en alimentación diaria, diferenció a las estrategias en dos grupos, gastos altos (estrategias tres y cuatro) y bajos (estrategias uno y dos). Los gastos en compra de concentrados representaron el $63,62,51$ y $76 \%$ de los gastos en alimentación para las estrategias uno, dos, tres y cuatro, respectivamente. $\mathrm{Si}$ bien este estudio no logró abordar el proceso de toma de decisiones en el diseño de las estrategias de alimentación, cabe resaltar lo observado en la estrategia tres con el menor gasto en concentrados (Cuadro 5), una mayor participación de subproductos $46 \%$ (Cuadro 2) y el menor contenido de fibra $40 \%$ MS (Cuadro 3) en la composición de la dieta; esto debido a que a nivel de intensidad de 
emisiones, esta estrategia presentó la menor intensidad de emisiones de $\mathrm{CH}_{4}$ (18 g CH$/ \mathrm{kg} \mathrm{LCPG)} \mathrm{conjuntamente}$ con la estrategia cuatro. Esto hace inferir que el consumo de concentrado no determina una menor intensidad de emisiones en las lecherías costarricenses.

Cuando se realizó el análisis de los gastos directos por tipo de alimento en cada estrategia, se pudo identificar que los concentrados representaron más del $50 \%$ de los costos de alimentación en las estrategias uno, dos y cuatro, mientras que en la tres fue del $49 \%$ (Cuadro 5). La estrategia uno presentó el menor gasto en concentrados con 1,64 $( \pm 0,92)$ dólares/animal/día, aunque similar a las estrategias dos y tres, mientras que la cuatro presentó el costo más alto en consumo de concentrados, este fue superior a la estrategia uno en casi un 100\% (Cuadro 5). Los gastos en subproductos identificaron a la estrategia tres como diferente del resto de grupos, con 1,66 dólares/animal/día. Los gastos en heno y ensilaje no presentaron diferencias significativas entre estrategias, al igual que los gastos incurridos en fertilización de pastos de piso. En el caso de los gastos por fertilización de pastos de corte, este diferenció a la estrategia dos (mayores gastos), de las estrategias uno y cuatro. La estrategia tres presentó gastos similares a todas las estrategias (Cuadro 5).

Este estudio permitió identificar cuatro diferentes estrategias de alimentación en altitudes entre 100 hasta los $2400 \mathrm{msnm}$. Un estudio realizado por Baars (1998) en fincas lecheras en Costa Rica, encontró que los gastos en alimentación de vacas en producción, representaban el $31 \%$ de los ingresos por venta de leche para la zona de mayor altitud, mientras que para la zona de baja altitud, estos gastos representaron el $26,5 \%$. Las lecherías de bajuras tienden a ser sistemas menos intensivos, así lo demuestra la estrategia uno perteneciente al rango altitudinal menor 383 $( \pm 410) \mathrm{msnm}$, la cual presentó la mayor intensidad de emisiones para la producción de leche $(0,68 \mathrm{~kg} \mathrm{CO}$ eq/ kg LCPG), con una mayor participación de pastos de piso y un mayor contenido de fibras en la dieta (Cuadro 2 y 3). Existe una evidente complejidad para abordar las estrategias de mitigación, debido a una relación entre variables endógenas (e.g factores culturales, genética animal, fuentes forrajeras presentes en el lugar) y exógenas (e.g variables climáticas, geografía de entorno, conectividad vial y variaciones de precios

Cuadro 5. Gastos de alimentos e ingresos sobre gastos de alimentación (IOFC) en las fincas lecheras asociadas a la Cooperativa Dos Pinos R.L. y basadas en conglomerados en función del porcentaje de representatividad de cada tipo de alimento en la dieta, áreas destinadas a potreros y pastos de corte, y el tiempo que los animales pasan diariamente en potreros. Costa Rica. 2014.

Table 5. Feed costs and income over feed costs (IOFC) in dairy farms affiliated to Dos Pinos Cooperative grouped in clusters based on the percentage of type of food in the diet, areas of forage and cut and carry pastures, and the daily time animals spend in pastures. Costa Rica. 2014.

\begin{tabular}{lccccc}
\hline Variable & \multicolumn{4}{c}{ Conglomerado } & P \\
\cline { 2 - 4 } & $\mathbf{1}$ (D.E.) & 2(D.E.) & 3(D.E.) & 4(D.E.) & \\
\hline Gastos (US dólares/día) & & & & \\
Concentrado & $1,64^{\mathrm{B}}( \pm 0,92)$ & $2,04^{\mathrm{B}}( \pm 1,05)$ & $2,30^{\mathrm{B}}( \pm 0,78)$ & $3,36^{\mathrm{A}}( \pm 0,84)$ & $<0,001$ \\
Subproductos & $0,19^{\mathrm{B}}( \pm 0,22)$ & $0,23^{\mathrm{B}}( \pm 0,50)$ & $1,66^{\mathrm{A}}( \pm 1,40)$ & $0,43^{\mathrm{A}}( \pm 0,30)$ & $<0,001$ \\
Ensilajes y heno & $0,14( \pm 0,42)$ & $0,38( \pm 1,21)$ & $0,41( \pm 0,73)$ & $0,18( \pm 0,31)$ & $\mathrm{NS}$ \\
Fertilización pastos piso & $0,47^{( \pm 0,36)}$ & $0,55( \pm 0,71)$ & $0,27( \pm 0,23)$ & $0,39( \pm 0,37)$ & $\mathrm{NS}$ \\
Fertilización pastos de corte & $0,0043^{\mathrm{B}}( \pm 0,01)$ & $0,06^{\mathrm{A}}( \pm 0,14)$ & $0,04^{\mathrm{AB}}( \pm 0,06)$ & $0,02^{\mathrm{B}}( \pm 0,05)$ & $<0,001$ \\
Total gastos alimentación & $2,45^{\mathrm{B}}( \pm 1,16)$ & $3,28^{\mathrm{B}}( \pm 2,07)$ & $4,68^{\mathrm{A}}( \pm 1,86)$ & $4,44^{\mathrm{A}}( \pm 1,06)$ & $<0,001$ \\
Ingresos ${ }^{1}$ (US dólares/día) & $7,44^{\mathrm{B}}( \pm 2,24)$ & $8,33^{\mathrm{B}}( \pm 2,74)$ & $8,29^{\mathrm{AB}}( \pm 2,18)$ & $9,80^{\mathrm{A}}( \pm 2,94)$ & $<0,1$ \\
IOFC $^{2}$ (US dólares vaca/día) & $4,99( \pm 1,67)$ & $5,05( \pm 2,56)$ & $3,62( \pm 1,31)$ & $5,36( \pm 2,51)$ & $\mathrm{NS}$ \\
\hline
\end{tabular}

\footnotetext{
${ }^{1}$ Ingresos por venta de leche / Income from milk sales

${ }^{2}$ Ingreso sobre gastos de alimentación / Income over feed cost.

A, B: Prueba Kruskal-Wallis, valores que no comparten el mismo superíndice son diferentes $(\mathrm{P}<0,5) /$ Kruskal-Wallis test; values within a row that do not share a superscript letter differ $(\mathrm{P}<0.05)$.
} 
de los insumos) que afectan al sistema y por ende las distintas fuentes de emisiones de GEI en la producción de leche.

En promedio el $70 \%$ de las emisiones dentro de la finca provino del $\mathrm{CH}_{4}$ por fermentación entérica, emisiones que varían respecto a las distintas estrategias de alimentación identificadas. Además, las estrategias de alimentación variaron espacialmente (i.e lecherías de bajura y lecherías de altura), por lo tanto, el objetivo de mitigación de la NAMA en ganadería tiene un subnivel que debe ser tomado en consideración a nivel regional. Los productores que componen la estrategia uno tienen una fuerte dependencia de pastos de piso los cuales muy posiblemente se verán afectados por los escenarios climáticos futuros, especialmente por las disminuciones graduales en precipitación; esto conllevará a esfuerzos económicos de magnitudes distintas para el financiamiento de los cambios tecnológicos necesarios para disminuir vulnerabilidades en época seca, donde la disponibilidad y la calidad nutricional de los pastos disminuyen, aumentando las emisiones de $\mathrm{CH}_{4}$ principal componente de emisiones de GEI en este estudio.

Para los productores que componen la estrategia tres y cuatro su dependencia se basó en los subproductos (cáscara de piña, caña y desechos orgánicos) y concentrados. La sostenibilidad de estas estrategias en el tiempo dependerá de la disponibilidad y los precios de los insumos externos. Los precios de la soya y maíz tienen un comportamiento errático y altamente sensible a las decisiones de países como Brasil, Argentina y Estados Unidos. La implementación de un NAMA en ganadería requiere de procesos subnacionales para determinar las necesidades reales y los incentivos adecuados para el fortalecimiento de la estrategia de ganadería baja en emisiones. Los requerimientos tecnológicos y cambios en las prácticas de manejo orientados a la mitigación, no serán los mismos entre las lecherías especializadas de bajura respecto a las de altura. El proceso transformacional solo se dará si existe una cohesión entre incentivos basados en mercados (certificaciones de huella de carbono, bonos de carbono, créditos verdes) con un marco institucional estable que genere confianza al sector privado sobre las inversiones en tecnologías orientadas a la mitigación y/o compensación de GEI.

\section{AGRADECIMIENTOS}

Los autores agradecen la contribución de Hector Léon Hidalgo (Cooperativa Dos Pinos) por la ayuda proporcionada en el acceso y recopilación de información de la base de datos de la cooperativa.

\section{LITERATURA CITADA}

Amador, M., J.C. Rodríguez, y A. Arroyo. 2010. Dinámica del rendimiento y digestibilidad del king grass en tres frecuencias de corte. Tierra Trop. 6:63-69.

Baars, R. 1998. Nutrition management, nitrogen efficiency, and income over feed cost on dairy farms in Costa Rica. J. Dairy Sci. 81:801-806.

Balzarini, M.G., L. Gonzalez, M. Tablada, F. Casanoves, J.D. Rienzo, y C.W. Robledo. 2008. Manual de usuario, versión 2008. Editorial Brujas, Córdoba, ARG.

Bannink, A., J.L. Ellis, N. Mach, J.W. Spek, and J. Dijkstra. 2013. Interactions between enteric methane and nitrogen excretion in dairy cows. Advances in Anim. Biosci. 4:19-27. doi: 10.1017/S2040470013000277

Bargo, F., L.D. Muller, J.E. Delahoy, and T.W. Cassidy. 2002. Performance of high producing dairy cows with three different feeding systems combining pasture and total mixed rations. J. Dairy Sci. 85:2948-2963. doi: 10.3168/jds.S0022-0302(02)74381-6

Buza, M.H., L.A. Holden, R.A. White, and V.A. Ishler. 2014. Evaluating the effect of ration composition on income over feed cost and milk yield. J. Dairy Sci. 97:30733080. doi: 10.3168/jds.2013-7622.

Chacón, A.R., J. Montenegro, y J. Sasa. 2009. Inventario nacional de gases con efecto invernadero y absorción de carbono en Costa Rica en el 2000 y 2005. Instituto Metereológico Nacional, San José, CRC.

Chacón-Hernández, P.A., y C.F. Vargas-Rodríguez. 2010. Consumo de Pennisetum purpureum cv. King Grass a tres edades de cosecha en caprinos. Agron. Mesoam. 21:267-274.

Conover, W.J. 1999. Practical nonparametric statistics. John Wiley \& Sons, Inc., NY, USA.

Cottle, D., J. Nolan, and S. Wiedemann. 2011. Ruminant enteric methane mitigation: a review. Anim. Prod. Sci. 51:491-514.

Dong, H., J. Margino, T.A. McAllister, J.D. Hatfield, D.E. Johnson, K.R. Lassey, M.A. de Lima, A. 
Romanovskaya, D. Bartram, D. Gibb, and J.H. Jr. Martin. 2006. Emission from livestock and manure management. In: S. Eggleston et al., editors, 2006 IPCC Guidelines for national greenhouse gas inventories. Vol 4, Agriculture, forestry and other land use. Intergovernmental panel on Climate Change, Hayacama, JPN. p. 10.7-10.82

Di Rienzo J.A., F. Casanoves, M.G. Balzarini, L. González, M. Tablada, y C.W. Robledo. 2014. InfoStat, versión 2008. Grupo InfoStat, FCA, Universidad Nacional de Córdoba, ARG.

FAOSTAT. 2014. Food and Agriculture Organization of the United Nations. Statistical Database. http:// faostat3.fao.org/browse/R/RL/S (Consultado 13 de junio 2014).

Gollnow, S., S. Lundie, A.D. Moore, J. McLaren, N. van Buuren, P. Stahle, K. Christie, D. Thylmann, and T. Rehl. 2014. Carbon footprint of milk production from dairy cows in Australia. Int. Dairy J. 37:31-38. doi: 10.1016/j.idairyj.2014.02.005

Hardie, C.A., M. Wattiaux, M. Dutreuil, R. Gildersleeve, N.S. Keuler, and V.E. Cabrera. 2014. Feeding strategies on certified organic dairy farms in Wisconsin and their effect on milk production and income over feed costs. J. Dairy Sci. 97:4612-4623. doi:10.3168/jds.20137763

Houghton, J.T., L.L. Meira Filho, B.A. Callander, N. Harris, A. Kattenberg, and K. Maskell. 1996. Climate change 1995. The science of climate change. Cambridge University Press, Cambridge, GBR.

IDF (International Dairy Federation). 2010. A common carbon footprint approach for dairy: The IDF guide to standard lifecycle assessment methodology for the dairy sector. International Dairy Federation, Brussels, BEL

IMN (Instituto Metereológico Nacional). 2014. Factores de emisión de gases efecto invernadero. San José, CRC.

Jiménez-Castro, J.P., y J.A. Elizondo-Salazar. 2014. Balance de nitrógeno en fincas para la producción de leche en Costa Rica. Agron. Mesoam. 25:151-160.

Johnson, K.A., and D.E. Johnson. 1995. Methane emissions from cattle. J. Animal Sci. 73:2483-2492.

Madriz, J.A. 2013. Situación actual y perspectivas del sector lácteo costarricense: visión de la Cámara Nacional de Productores de Leche. Comunicación presentada en: XX Congreso Nacional lechero. 4-5 dic. San Carlos, CRC

MAG (Ministerio de Agricultura y Ganadería). 2013. Concepto NAMA fincas ganaderas. MAG, San José, CRC
Mata, L. 2011. Tabla de composición de materias primas usadas en alimentos para animales. Centro de Investigaciones en Nutrición Animal (CINA), San José, CRC.

MIDEPLAN (Ministerio de Planificación Nacional y Política Económica). 2010. Plan Nacional de Desarrollo 20112014. "María Teresa Obregón Zamora", MIDEPLAN, San José, CRC

MINAET (Ministerio de Ambiente, Energía y Telecomunicaciones). 2009. Estrategia nacional de cambio climático. Editorial Calderón y Alvarado S.A., San José, CRC.

Montenegro, J., y S. Abarca. 2001. Importancia del sector agropecuario costarricense en la mitigación del calentamiento global. MAG/IMN, San José, CRC.

Mora, C.V. 2001. Fijación, emisión y balance de gases de efecto invernadero en pasturas en monocultivo y en sistemas silvopastoriles de fincas lecheras intensivas de las zonas altas de Costa Rica. Tesis MSc, CATIE, Turrialba, CRC.

Mora, M.A., y C.B. Figueroa. 2005. Producción de forraje y calidad nutricional de variedades de Pennisetum purpureum en la Meseta Central de Costa Rica. Agron. Mesoam. 16:37-43.

NRC (National Research Council). 2001. Nutrient requirements of dairy cattle. 7 rev. ed. 2001. The National Academies Pres, USA.

Oenema, O., N. Wrage, G. Velthof, J.W. Groenigen, J. Dolfing, and P. Kuikman. 2005. Trends in global nitrous oxide emissions from animal production systems. Nutr. Cycl. in Agroecosyst. 72:51-65. doi: 10.1007/s10705-004-7354-2

Ortiz, M.E. 2009. Atlas digital de Costa Rica 2008. CDRom. Instituto Tecnológico de Costa Rica, Cartago, CRC.

Patel, M., E. Wredle, G. Börjesson, R. Danielsson, A.D. Iwaasa, E. Spörndly, and J. Bertilsson. 2011. Enteric methane emissions from dairy cows fed different proportions of highly digestible grass silage. Acta Agriculturae Scandinavica: Section A. Anim. Sci. 61:128-136. doi:10.1080/09064702.2011.616216.

Sánchez, J.M., L. Piedra, y H. Soto. 1998. Calidad nutricional de los forrajes en zonas con niveles bajos de producción de leche, en la zona norte de Costa Rica. Agron. Costarricense 22(1):69-76.

Sánchez, J.M., y H. Soto. 1996. Estimación de la calidad nutricional de los forrajes del cantón de San Carlos. I. Materia seca y componentes celulares. Rev. Nutr. Anim. Trop. (Costa Rica) 3(1):3-18. 
Sánchez, J.M., y H. Soto. 1999a. Calidad nutricional de los forrajes de una zona con niveles medios de producción de leche en el trópico húmedo del norte de Costa Rica. Agron. Costarricense 23(2):165-171.

Sánchez, J.M., y H. Soto. 1999b. Niveles de energía estimada en los forrajes de un distrito de mediana producción lechera, Fortuna de San Carlos, en la zona norte de Costa Rica. Agron. Costarricense 23(2):179-185.

Schils, R.L.M., J. Eriksen, S.F. Ledgard, T.V. Vellinga, P.J. Kuikman, J. Luo, S.O. Petersen, and G.L. Velthof. 2013. Strategies to mitigate nitrous oxide emissions from herbivore production systems. Animal 7(Supplements1):29-40. doi:10.1017/ S175173111100187X

Scott, R. 1986. Cheesemaking practice. 2 ed. Springer Science, USA.

Sehested, J., T. Kristensen, and K. Søegaard. 2003. Effect of concentrate supplementation level on production, health and efficiency in an organic dairy herd. Livest. Prod. Sci. 80:153-165. doi:10.1016/S03016226(02)00317-2

SEPSA (Secretaría Ejecutiva de Planificación Sectorial Agropecuaria). 2013. Política de estado para el sector agroalimentario y el desarrollo rural costarricense 2010-2021. SEPSA/MAG. San José, CRC.

Soto, C., A. Valencia, R.D. Galvis, and H.J. Correa. 2005. Effects of the cutting age and the level of nitrogen fertilization over the energetic and proteic value of the grass 'kikuyo' (Pennisetum clandestinum). Rev. Colom. Cienc. Pecu. 18:17-26.
Van Horn, H., A. Wilkie, W. Powers, and R. Nordstedt. 1994. Components of dairy manure management systems. J. Dairy Sci. 77:2008-2030.

Van Middelaar, C.E., P.B.M. Berentsen, J. Dijkstra, J.A.M. van Arendonk, and I.J.M. de Boer. 2014. Methods to determine the relative value of genetic traits in dairy cows to reduce greenhouse gas emissions along the chain. J. Dairy Sci. 97:5191-5205. doi:10.3168/jds.2013-7413

Villalobos, L., J. Arce, y R. WingChing. 2013. Producción de biomasa y costos de producción de pastos estrella africana (Cynodon nlemfuensis), kikuyo (Kikuyuocloa clandestina) y ryegrass perenne (Lolium perenne) en lecherías de Costa Rica. Agron. Costarricense 37(2): 91-103.

Villalobos, L., y J.M. Sánchez. 2010. Evaluación agronómica y nutricional del pasto ryegrass perenne tetraploide (Lolium perenne) producido en lecherías de las zonas altas de Costa Rica. II. Valor nutricional. Agron. Costarricense 34(1):43-52.

Villarreal, M. 1992. Evaluación comparativa de ratana (Ischaemum ciliare) como especie forrajera. Agron. Costarricense 16(1):37-44.

Wolf, C.A. 2010. Understanding the milk-to-feed price ratio as a proxy for dairy farm profitability. J. Dairy Sci. 93:4942-4948. doi:10.3168/jds.2009-2998

Yan, T., C.S. Mayne, and M.G. Porter. 2006. Effects of dietary and animal factors on methane production in dairy cows offered grass silage-based diets. International Congress Series 1293:123-126. doi:10.1016/j. ics.2006.02.024 
